\title{
Non-Local Effects at the Onset of the Chiral Transition
}

\author{
Letícia F. Palhares ${ }^{1}$, Eduardo S. Fraga ${ }^{1}$, Takeshi Kodama ${ }^{1}$, and Gastão Krein ${ }^{2}$ \\ ${ }^{1}$ Instituto de Física, Universidade Federal do Rio de Janeiro \\ C.P. 68528, Rio de Janeiro, RJ 21941-972, Brazil \\ ${ }^{2}$ Instituto de Física Teórica, Universidade Estadual Paulista \\ Rua Pamplona 145, São Paulo, SP 01405-900, Brazil
}

Received on 19 October, 2006

\begin{abstract}
Inspired by analytic results obtained for a systematic expansion of the memory kernel in dissipative quantum mechanics, we propose a phenomenological procedure to incorporate non-markovian corrections to the Langevin dynamics of an order parameter in field theory systematically. In this note, we restrict our analysis to the onset of the evolution. As an example, we consider the process of phase conversion in the chiral transition.

Keywords: Memory effects; Langevin dynamics; Chiral phase transition
\end{abstract}

\section{INTRODUCTION}

For high enough values of temperature, strongly interacting matter should be in a quark phase due to asymptotic freedom. For the last few years, relativistic high-energy heavy ion collisions have been providing valuable information on the new state of matter that seems to have been created according to recent data from experiments at BNL-RHIC [1], even though its true nature is still uncertain. Moreover, comparison between temperature estimates from data and results from Lattice QCD apparently shows that the critical temperature $\left(T_{c} \sim 150 \mathrm{MeV}\right)$ for the chiral transition has been exceeded [2].

As the deconfined plasma created in a heavy-ion collision expands, it cools down, undergoing a phase transition (or a rapid crossover) back to hadronic matter, as well as breaking the approximate chiral symmetry. Results from CERNSPS and BNL-RHIC feature an explosive behavior in the hadronization process and seem to favor a very fast spinodal decomposition as the mechanism of phase conversion. Effective chiral field theory models for QCD also point to an explosive spinodal decomposition scenario $[3,4]$.

Under these conditions, since relevant time scales are not so clearly separated (as, for instance, in the case of the primordial quark-hadron transition), the competition between several dynamical phenomena will significantly affect the phase conversion process. In this context, fluctuations and non-local effects will play a major role in the dynamics of the order parameter. Therefore, a linear response non-equilibrium field theory approach will provide a highly non-local Langevin equation for the evolution of the chiral condensate [5], and the memory kernel details will presumably be important in the determination of the physical time scales for the whole conversion process [6]. Most likely one will find interesting transient effects and, eventually, a hierarchy of time scales.

Since the structure of memory integrals and colored noise that appear in field theory, even for relatively simple effective models, is often rather complicated, one has to resort to numerical simulations to obtain exact results [7]. Analytic results, which could bring some qualitative understanding of the mechanisms involved, can only be achieved within systematic approximations [8].

In this paper, we propose a phenomenological procedure to incorporate non-markovian corrections to the Langevin dynamics of an order parameter in field theory systematically. We follow a path inspired by analytic results obtained for a systematic expansion of the memory kernel in dissipative metastable quantum mechanics, a much simpler problem which we use as a toy model. To avoid some technical complications, we restrict our analysis to the onset of the evolution, i.e., to very early times. Moreover, for simplicity, we intentionally neglect inhomogeneity effects and the noise contribution, although we know they will be important and should be incorporated $[9,10]$. These issues, as well as a detailed comparison to exact (numerical) results, will be addressed in a future publication [8]. As an application, we consider the process of phase conversion in the chiral transition, comparing our results to those from a markovian Langevin evolution [6]. In view of the caveats mentioned above, our results for the initial dynamics of the order parameter should be taken as a hint to the complex transient regime that can only be unveiled by a more rigorous approach.

The paper is organized as follows. Section II presents the method in the framework of non-markovian dissipative quantum mechanics, giving special attention to the features that will be important in the phenomenological extension to field theory. The early-time dynamics of the chiral condensate with the first phenomenological non-local corrections is discussed in Section III. Section V contains our conclusions and outlook.

\section{NON-MARKOVIAN DISSIPATIVE METASTABLE QUANTUM MECHANICS}

The development of a systematic procedure to take nonlocal effects into account should have as starting point a careful determination of the domain of validity of the local approximation. However, as pointed out above, the structure of memory kernels that come about in non-equilibrium field-theoretic approaches is rather intricate. This may turn the determination of the domain of validity into an excessively complex task. To isolate the analysis of the role of non-locality, we consider a much simpler case in quantum mechanics (QM), where the problem can be solved in a detailed and controlled fashion.

Our QM toy model is constituted by a particle, with co- 
ordinate $q$ and momentum $p$, interacting linearly with a heat bath which introduces dissipation in the dynamical evolution. The reservoir is modeled in the usual Caldeira-Leggett fashion [11]. The hamiltonian is thus given by:

$$
\begin{aligned}
H & =\frac{p^{2}}{2 M}+V(q)+H_{R}+\theta(t) q \sum_{k} c_{k} R_{k} ; \\
H_{R} & =\sum_{k}\left(\frac{p_{k}^{2}}{2 m}+\frac{1}{2} m \omega_{k}^{2} R_{k}^{2}\right),
\end{aligned}
$$

where the sum over the heat bath modes is limited by a "cutoff" $\Omega$, that corresponds to the maximum frequency for the interaction of the system with the medium. We also assume the system to be suddenly immersed into the reservoir at $t=0$ (which represents a rough simulation of the system "quench" in the chiral transition), so that the approximation of an initially uncorrelated density matrix is reasonable:

$$
\rho(t \leq 0)=(|0\rangle\langle 0|)_{q} \otimes e^{-\beta H_{R}},
$$

where $T=1 / \beta$ is the reservoir temperature.

The evolution towards equilibrium is studied within the Schwinger-Keldysh closed-time path framework [12]. Integrating over the bath variables, we obtain the effective action. After performing a Wigner transform to separate the classical coordinate, $Q(t)$, from the fluctuations (associated with a Gaussian noise $\xi(t)$ ) the equation of motion assumes the following semiclassical generalized Langevin form:

$$
\begin{array}{r}
M \ddot{Q}+\bar{V}^{\prime}(Q)+\frac{2 \eta}{\pi} \int_{0}^{t} d t^{\prime} \frac{\sin \left[\Omega\left(t-t^{\prime}\right)\right]}{t-t^{\prime}} \dot{Q}\left(t^{\prime}\right)=\xi(t) \\
\left\langle\xi(t) \xi\left(t^{\prime}\right)\right\rangle=\frac{2 \eta T}{\pi} \frac{\sin \left[\Omega\left(t-t^{\prime}\right)\right]}{t-t^{\prime}}
\end{array}
$$

where $\eta$ is a phenomenological dissipation parameter encoding information about the heat bath and $\bar{V}^{\prime}(Q)$ is the derivative with respect to $Q$ of the modified particle potential,

$$
\bar{V}^{\prime}(Q) \equiv V^{\prime}(Q)-[2 \eta \Omega / \pi] Q
$$

In the markovian limit, this correction represents an ultraviolet divergence that is associated with the unphysical situation in which the particle interacts with all frequencies of the heat bath. Usually, in the context of quantum dissipative systems, this alteration in the potential is ignored, under the argument that the interest resides on the modifications brought about by dissipation and not by medium renormalization effects. In quantum field theory, however, the "particle" and the reservoir are deeply entangled, corresponding to different Fourier modes of the same field (refer to [8] for further discussion). Since we are ultimately interested in the chiral transition case, we compare in this analysis the evolutions with and without the modification in the potential.

The limit in which $\Omega$ is strictly infinite yields a local dissipation term in Eq. (4). Therefore, the non-markovian contributions arise from the assumption that $\Omega$ is still large, but finite. As one goes down in $\Omega$ one should, in principle, include more and more non-local corrections. Taking advantadge of the localized profile of the kernel that appears in Eq. (4) and assuming that the system will eventually thermalize in a stable vacuum, we can derive the following series for the memory integral (for details, see [8]):

$$
M \ddot{Q}+\bar{V}^{\prime}(Q)+\frac{2 \eta}{\pi} \sum_{n=0}^{\infty} \frac{J_{n}(\Omega t)}{n !} \frac{Q^{(n+1)}(t)}{\Omega^{n}}=\xi(t),
$$

where the time-dependent coefficients $J_{n}(\Omega t)$ contain information about the specific form of the original kernel. It should be noticed that the first term corresponds to the usual markovian approximation, in the limit $\Omega \rightarrow \infty$.

The oscillatory behavior of the integrand in Eq. (4) is inherited by the $J_{n}$ 's. Nevertheless, $J_{0}$ and $J_{1}$ have definite signs: $J_{0}>0$ and $J_{1} \leq 0$. However, for $n \geq 3$, one can show that the signs of those coefficients change according to the time at which it is being evaluated. Even though the amplitudes of the associated terms decrease, rendering these oscillations irrelevant for long times, the transient dynamics will be greatly affected.

We remark that the localized profile of the memory kernel is the essential feature for the development of the nonlocal series described above. The oscillatory characteristic of the $J_{n}$ 's is a specificity of the function $\sin (x) / x$ and its consequences do not encode modifications due to non-locality. Therefore, the alternating signs should not be interpreted as intrinsic memory effects. As a matter of fact, the essential feature being added here, as compared to the markovian case, is a non-zero correlation for times separated by an interval $\Delta t \lesssim 1 / \Omega$. For $\Omega$ large enough, higher-order contributions in Eq. (6) become more and more negligible and the series can be truncated. This procedure systematically incorporates the effects from correlations between times within this interval.

Now we analyze the non-local Langevin evolution dictated by Eq. (6) for the double-well potential, namely for $V^{\prime}(Q)=$ $4 \lambda Q^{3}-M \omega_{0}^{2} Q$, at early times. In this regime, the equation is linear. For simplicity, we also approximate the coefficients by constants. Keeping terms up to the third-order time derivative, one has:

$$
\begin{gathered}
\bar{M} \ddot{Q}-\left[M \omega_{0}^{2}+2 \eta \Omega / \pi\right] Q+\eta \dot{Q}+\eta_{3} \dddot{Q}=\xi(t) \\
\bar{M}=M\left(1+\frac{2}{\pi} \frac{\eta}{M \Omega} J_{1}\right) \quad, \quad \eta_{3}=\frac{2}{\pi} \frac{\eta}{\Omega^{2}} J_{2}
\end{gathered}
$$

The solution of Eq. (7) is obtained through a straightforward calculation, using the Laplace transform. Results for the average $\langle Q\rangle$ as a function of time are displayed in Fig. 1, for $\langle\dot{Q}(0)\rangle=\langle\ddot{Q}(0)\rangle=0$. One can see that both memory and potential corrections tend to accelerate the initial decay process towards the minimum at $Q=Q_{v a c}$. It is clear that the modification of the potential introduces the major effect, remarkably reducing the time scale involved in the decay process. Although this correction may be ignored in the analysis of dissipative phenomena in QM, as discussed previously, it must be taken into account in the field theory case. In the latter, the medium renormalization of the effective potential should play a significant role in the phase transition process.

The fact that not only the medium modification of the potential but also the memory correction tend to speed up the 


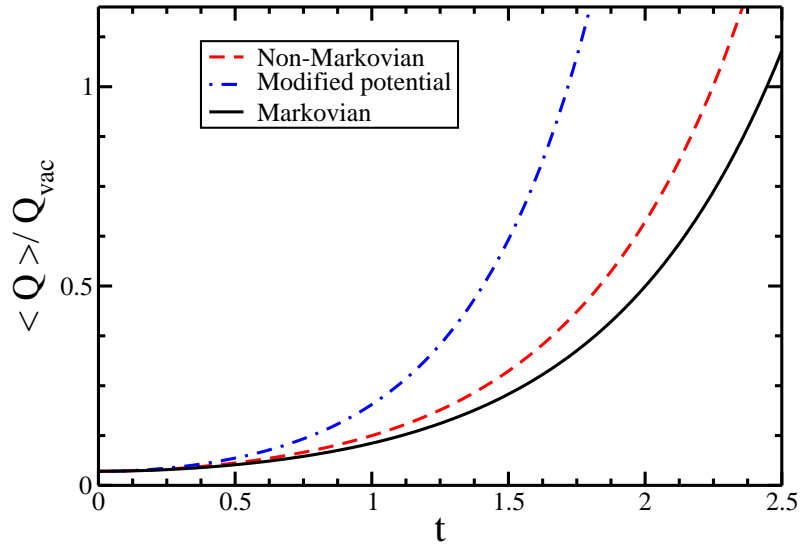

FIG. 1: The average of $Q$ in units of its vacuum value as a function of time for $M=\omega_{0}=\eta=2$ in arbitrary units and $\langle Q(0)\rangle=0.04 Q_{v a c}$. The dashed curve corresponds to the non-markovian case with $\Omega=$ $2 \Lambda \approx 3.852$, while the dot-dashed one is the non-local solution including the potential renormalization for the same value of $\Omega$.

decay of the particle seems surprising at first sight. In fact, one expects the latter to slow down the rollover process. The behavior observed in Fig. 1 is certainly a transient effect and should be washed out in the long run by dissipation and nonlinear effects [8]. Nevertheless, it points to the possibility of a non-trivial transient regime at early times. We will return to this point in the next section.

\section{CHIRAL TRANSITION EARLY-TIME DYNAMICS}

Inspired by the results obtained above for the QM toy model, we can construct a phenomenological generalization to the case of field theory. In particular, we consider the case of the chiral transition. The effective potential, $U_{\text {eff }}(\phi, T)$, for the chiral condensate $\phi(\vec{x}, t)$ can be obtained, for instance, from an effective chiral model as described in Ref. [4].

The local form of the Langevin dynamics for the chiral condensate, which plays the role of a non-conserved order parameter in a Landau-Ginzburg approach, can be derived under convenient approximations within the framework of nonequilibrium field theory [5], and is given by

$$
\square \phi+\Gamma(T) \frac{\partial \phi}{\partial t}+U_{e f f}^{\prime}(\phi, T)=\xi(\vec{x}, t) .
$$

The second term in Eq. (9) corresponds to markovian dissipation and the right-hand side represents a gaussian, white stochastic force, both attained, in principle, after drastic approximations of complicated memory kernels [5]. Some of us have recently analyzed the role of dissipation in the hadronization of the quark-gluon plasma under these conditions [6].

The inclusion of non-local effects is done, phenomelogically, by inserting higher-order time derivatives of $\phi(\vec{x}, t)$ into
Eq. (9):

$$
\square \phi+\Gamma \frac{\partial \phi}{\partial t}+\Gamma_{2} \frac{\partial^{2} \phi}{\partial t^{2}}+\Gamma_{3} \frac{\partial^{3} \phi}{\partial t^{3}}+U_{e f f}^{\prime}(\phi, T)=\xi(\vec{x}, t) .
$$

Trying to follow the scale hierarchy that arises naturally in the series expansion in QM, we choose the form of the coefficients accompanying these new terms. In addition to being proportional to the phenomenological temperature-dependent dissipation coefficient $\Gamma$, which can be estimated using kinetic theory, the $\Gamma_{n}$ 's are associated with increasing inverse powers of a large scale $\Omega$ : $\Gamma_{n}(T)=(-1)^{n} \Gamma / \Omega^{n-1}$. Here, $\Omega$ is the parameter that controls the revelance of non-locality and imposes the intended hierarchy between the contributions of different orders of time derivatives. For the above truncation of the series that presumably appears in Eq. (10) to be valid, $\Omega$ must be large (although finite) compared to the temperature $T$, the natural scale of the system. Since we focus on non-local corrections in time, $\Omega$ is the only relevant scale brought about by the kernel. In a more realistic description of the chiral transition, however, other scales must be taken into account, e.g. those associated with inhomogeneities [9].

To obtain analytical solutions of Eq. (10), one can resort to a sixth-order polynomial fit of $U_{e f f}(\phi, T)$ [6] and investigate the short-time dynamics. Since we consider the explosive spinodal decomposition scenario, the potential is assumed to be time-independent. Its form is fixed at the spinodal temperature $\left(T_{s p} \sim 108 \mathrm{MeV}\right)$, and the system is initially slightly displaced from the inflexion point $\left(\phi_{0} \sim 0.162 T_{s p}\right)$.

For short times, $U_{e f f}^{\prime}$ can be approximated by its linear form around $\phi_{0}$. We treat, for simplicity, the dynamics of the mean value of the field over the stochastic noise for a homogeneous initial condition. Therefore, Eq. (10) becomes equivalent to an infinite set of linear ordinary differential equations: each Fourier mode of the field evolves according to an analogue of Eq. (7). When one examines the solution averaged over space, one verifies that the only relevant mode is $k=0$. Under these conditions, the field theory case is reduced to a $\mathrm{QM}$ problem for the Fourier component $\phi_{k=0} \equiv \varphi$ :

$$
\begin{gathered}
\ddot{\varphi}+a \dot{\varphi}+b \dddot{\varphi}+c \varphi=0, \\
a=\frac{\Gamma}{1-\Gamma / \Omega} ; b=\frac{\Gamma}{\Omega^{2}(1-\Gamma / \Omega)} ; c=\frac{-U_{e f f}^{\prime \prime}\left(\phi_{0}\right)}{1-\Gamma / \Omega} .
\end{gathered}
$$

In Fig. 2, we present results for the initial dynamics of the order parameter $\phi(\vec{x}, t)$ subject to a non-markovian Langevin evolution. The behavior at times larger than those shown in the figure reproduces qualitatively the QM case, Fig. 1.

The existence of a competition between effects from different memory corrections is illustrated in Fig. 2. Although these results are valid only for short times, the study of these curves enlightens the role of each term in the dynamics of the phase conversion.

The early influence of non-local dissipation is to retard the phase transition process. At extremely short times, the lowerorder time derivatives are subdominant, so that $\varphi \approx e^{\lambda t}$ with $\lambda \approx(-c / b)^{1 / 3} \propto \Omega^{2 / 3}$. Thus, the outcome of smaller values of $\Omega$ will be a greater delay in the initial evolution. On the other hand, for intermediate times, the modification brought 


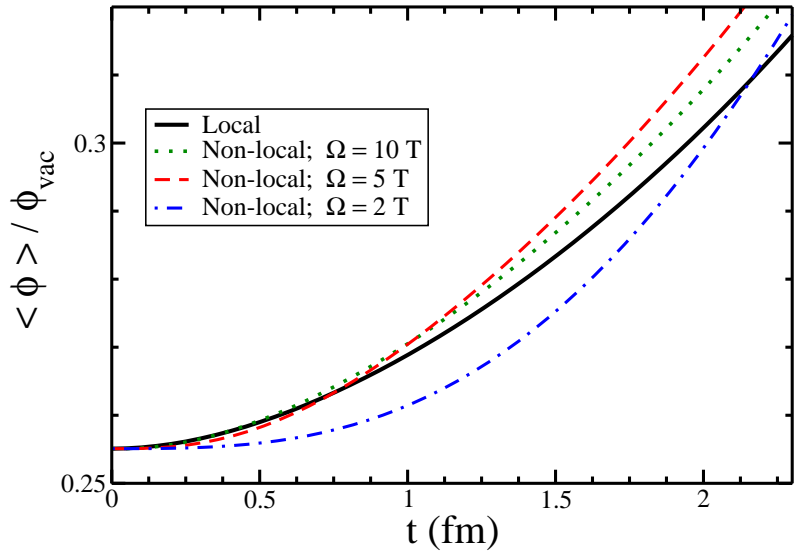

FIG. 2: The evolution at $T=T_{s p}$ of the average value of the order parameter over noise and space in units of its vacuum value. In addition to the local evolution (full line), three curves with non-markovian corrections are shown: $\Omega / T=10,5$ and 2 .

about by the third-order derivative is overcome by the nonlocal correction of the potential. In this case, as $\Omega$ increases, the exponent $\lambda$ will decrease, $\lambda \approx(-c)^{1 / 2}$, and the curves tend to the markovian one.

Features that were neglected in this study should play a major role in the transition dynamics, possibly washing out those transient effects observed here. We will discuss the main shortcomings of the method in our conclusion.

\section{CONCLUSIONS AND OUTLOOK}

We constructed a phenomenological generalization of the Langevin dynamics experienced by an order parameter in field theory, accounting for non-markovian correlations in a systematic fashion. We used as inspiration a toy model in dissipative metastable QM, where the domain of validity of the local approximation was identified, allowing us to derive a derivative expansion of the memory kernel. In this series, the first term corresponds to the usual markovian assumption and higher-order time derivatives are accompanied by increasing powers of the scale $1 / \Omega$, associated with the maximum time interval in which correlations exist.

It should be noticed that this systematic expansion method developed can not be applied to any memory kernel. In fact, the kernel must have a localized profile, so that correlations between sufficiently distant times vanish. This is in general not true when one considers a semiclassical expansion around a non-trivial background as, for instance, in the case of dynamical viscosity in nucleation [13].

Results presented for the onset of the non-markovian evolution of the chiral condensate neglecting stochastic noise effects revealed an intricate transient structure caused by the competition between different contributions. The influence of non-locality at extremely short times is to delay the transition process through a dissipation term proportional to a higher-order derivative of the field. At intermediate times, non-markovian modifications of the potential dominate the evolution, accelerating it.

In this work, we identified the role of each non-local correction in a very simple model for the early dynamics. The complete evolution will be studied numerically in a future publication [8]. In a more realistic approach, however, several other phenomena should be taken into account and an even more complex balancing will dictate the evolution towards the new vacuum state. The inclusion of the complete non-linear potential, for instance, would accelerate the early evolution and slow down the process for larger times, smoothening or even erasing the initial modifications observed above. The effect of stochastic noise should be considered, as well as inhomogeneities in the effective potential. Not only temporal, but also spatial non-locality should be taken into account. All these aspects could, in principle, affect significantly the time scales of the phase conversion.

To obtain reliable quantitative results in the field theory case, one must firstly derive this phenomenological non-local Langevin equation through non-equilibrium quantum field theory. In this procedure, the medium renormalization of the potential (which provides significant corrections in the QM case) would be incorporated naturally and the scale $\Omega$ could be identified and, possibly, estimated.

This work was partially supported by CAPES, CNPq, FAPERJ, FAPESP and FUJB/UFRJ.
[1] Proceedings of Quark Matter 2004, J. Phys. G 30, S633 (2004).

[2] E. Laermann and O. Philipsen, Ann. Rev. Nucl. Part. Sci. 53, 163 (2003).

[3] O. Scavenius and A. Dumitru, Phys. Rev. Lett. 83, 4697 (1999); O. Scavenius, A. Dumitru, and A. D. Jackson, Phys. Rev. Lett. 87, 182302 (2001); A. Dumitru and R. D. Pisarski, Nucl. Phys. A 698, 444 (2002).

[4] O. Scavenius et al., Phys. Rev. D 63, 116003 (2001).

[5] M. Gleiser and R. O. Ramos, Phys. Rev. D 50, 2441 (1994); C. Greiner and B. Muller, Phys. Rev. D 55, 1026 (1997); D. H. Rischke, Phys. Rev. C 58, 2331 (1998); Z. Xu and C. Greiner, Phys. Rev. D 62, 0360012 (2000).
[6] E. S. Fraga and G. Krein, Phys. Lett. B 614, 181 (2005).

[7] A. Arrizabalaga, J. Smit, and A. Tranberg, Phys. Rev. D 72, 025014 (2005); J. Berges and I. O. Stamatescu, Phys. Rev. Lett. 95, 202003 (2005).

[8] E. S. Fraga, T. Kodama, G. Krein, and L. F. Palhares, to appear.

[9] B. G. Taketani and E. S. Fraga, Phys. Rev. D 74, 085013 (2006).

[10] E. S. Fraga, Eur. Phys. J. A 29, 123 (2006).

[11] A. O. Caldeira and A. J. Leggett, Phys. Rev. Lett. 46, 211 (1981); Annals Phys. 149, 374 (1983).

[12] N. P. Landsman and C. G. van Weert, Phys. Rept. 145, 141 (1987).

[13] S. Alamoudi et al., Phys. Rev. D 60, 125003 (1999). 